\title{
Antiviral and anti-inflammatory effects of medicated thread moxibustion combined with Shujin Jiannao granule in the treatment of HFMD
}

\author{
HUANG Yue-yan ${ }^{1 \#, T A N G ~ H a n-q i n g}{ }^{1}$, LI Qing-feng ${ }^{1}$, WEI Yi ${ }^{2}$, \\ LIANG Wei ${ }^{2}, \mathrm{HUANG} \mathrm{Ken}^{1}, \mathrm{LI}$ Xiao-hua',HUANG Yun- \\ feng ${ }^{1}, H U A N G$ Zhi-jing ${ }^{1}$
}

\author{
${ }^{1}$ Youjiang Medical University for Nationalities , Baise, China \\ ${ }^{2}$ Hainan Medical University, Haikou, China
}

\begin{abstract}
Objective: To investigate antiviral and anti-inflammatory effects of medicated thread moxibustion combined with Shujin Jiannao granule in the treatment of HFMD. Methods: A total of 124 children with HFMD were collected to divided into western medicine group (62 cases) and experimental group (62 cases) while 62 healthy children were enrolled to become normal control group. Western medicine group was treated according to conventional therapy while experimental group was treated with combined treatment of traditional Chinese medicine (TCM) and western medicine. To determine serum levels of S100B, NSE, CRP and WBC and observe the clinical effectiveness. Results: In the western medicine group the total effectiveness rate is $88.7 \%$ while it is $96.8 \%$ in the experimental group $(\mathrm{P}<0.0)$; the average disappearance time of symptoms are shorter in the experimental group than that in the western medicine group $(\mathrm{P}<0.05)$. Compared with the control group, the serum levels of S100B, NSE, CRP and WBC all rise in HFMD children $(\mathrm{P}<0.05$ or $\mathrm{P}<0.01)$. After treatment, the serum levels of $\mathrm{S} 100 \mathrm{~B}$, NSE, CRP and WBC reduce in both the experimental group and the western medicine group, when compared with that before treatment. The difference is statistically significant $(\mathrm{P}<0.05$ or $\mathrm{P}<0.01)$.

Conclusion: Medicated thread moxibustion combined with Shujin Jiannao granule has good antiviral and antiinflammatory effects and will provide alternative for the treatment of HFMD..
\end{abstract}

Keywords: Hand-foot-mouth disease; medicated thread moxibustion; treatment; research

\section{Introduction}

Hand-foot-mouth disease ( HFMD) is a common infections in infants. Typical signs are the herpes and maculopapule diabrosis on hands, feet and mouth. The central nervous system and respiratory system were damaged in some of the critical cases, with performances such as drowsiness, shock, headache, cough, vomiting, etc. It requires active and effective treatment. HFMD is known to be caused by intestinal virus infection. The common viruses are EV71 and CoxA $16^{[1]}$. The clinical prognosis is grim with the performances of continuous high fever, limb shaking, myoclonus, nystagmus, ataxia, eye movement disorder, meningeal stimulation, tendon reflex abnormality, dyspnea, purple, bloody frothy sputum, lungs rale, etc. Traditional Chinese medicine believes that HFMD and nervous system damage belong to damp-heat diseases with syndrome of consumption of fluid due to intense heat. "Benefiting liver and kidney and invigorable blood and muscle" are applied to the treatment on Chinese medicine. Zhuang medicine believes that HFMD and nervous system damage belong to qi and blood inequality.Medicated thread moxibustion is used for promoting blood circulation to remove blood stasis, dredging paralysis and relieving pain. The combined therapy of western medicine, medicated thread moxibustion and traditional Chinese medicine Shujin Jiannao granule are used for the treatment of HFMD in the text.

Copyright (C) 2018 HUANG Yue-yan et al.

doi: 10.24294/jpd.v2i2.153

EnPress Publisher LLC.This work is licensed under the Creative Commons Attribution-NonCommercial 4.0 International License (CC BY-NC 4.0).

http://creativecommons.org/licenses/ by/4.0/ 


\section{Material and Method}

\section{Cases included}

\section{Diagnostic criteria for western medicine}

The inclusion criteria is on the basis of The Technical guide for the diagnosis and treatment of HFMD(Ministry of Health 2010), the diagnosis criteria established by The Ministry of Health. The main performances are fever, maculopapule and herpes emerged on hands, feet, mouth and buttocks. Specific nucleic acid test is positive on EV71 or CoxA16.The clinical diagnosis is HFMD, with one of the performances:

Continuous fever ( $\geq 3$ days); (2) Limbs shaking, myoclonic, nystagmus, ataxia, eye movement disorder. The stimulation of the meningeal membrane, the tendon reflexes weaken or disappearance; (3) Difficulty in breathing, purple, bloody frothy foaming, lung rale, etc.

\section{Creterion of TCM syndrome differentiation typing}

It refers to The Guide for Diagnosis and Treatment of HFMD (2010, in accordance with the diagnosis criteria of deficiency of liver and kidney and Qi stagnation and blood stasis syndrome: Prone to fatigue, numbness of limbs, dry throat, insomnia and dreaminess, rib dull pain, waist and knee pain, tinnitus, tongue red, little furred tongue, chest and rib swelling, irritability, purple tongue nature or ecchymosis, uneven pulse, etc. ${ }^{[5]}$.

\section{Zhuang medicine symptom classification criterion}

It refers to the Zhuang medicine symptom classification criterion of Pan Chen etc. The child patients with fear of cold, fever, swelling, pain, impotence, numbness etc ${ }^{[3]}$.

\section{Exclusion criteria}

(1) Merged child patents with severe function impairment of heart, lung, liver and kidney. (2) In accordance with the critical illness of child patients in The Guide for Diagnosis and Treatment of HFMD (2010). (3) Allergic to medicated thread moxibustion or child patients with allergic constitution or skin easy burning. (4) The child patients intolerance with medicated thread moxibustion.

\section{General Material}

The object of research is 124 cases of child patients diagnosed with HFMD in pediatric department in hospital from January 2016 to April 2017. It groups to western medicine treatment group (western medicine group) by random number table. Sixty-two cases, thirty-four male cases and twenty-eight female cases. The ages are from eleven months to ten years old. The average age is $3.12 \pm 0.92$ and the average course of disease is $5.8 \pm 1.6$. Sixty-two cases in the combined treatment of traditional Chinese medicine(TCM) and western medicine group(the experimental group), with thirty-two male cases and thirty female cases. The ages are from ten months to eleven years old. The average age is $3.24 \pm 0.94$ and the average course of disease is $5.6 \pm 1$.3. There is no statistical significance compared with the gender, age and average course of disease of the child patients. This study has been approved by the children's guardians informed consents and hospital ethics committee.

\section{Method}

\section{Therapeutic method}

Western medicine group: According to the western medicine therapeutic schedule recommended by The guide for the diagnosis and treatment of HFMD(2010), (1) Pay attention to isolation, avoid cross infection and take proper rest, light diet, good oral and skin care and expectant treatment. (2) Take 10-15 mg/kg lebavirin intravenous injection each time per day and continue for six days. Child patients with bacterial infections are treated with antibiotics together. (3) Take 8 $\mathrm{mg} / \mathrm{kg}$ methyl prednisolone each time per day; Take $1 \mathrm{~g} / \mathrm{kg}$ immunoglobulin each time per day. If the symptoms and signs are not obviously fade for three days, can be used for another one to three days. For severe child patients, the dose of methylprednisolone is $20 \mathrm{mg} / \mathrm{kg}$ each time, and the dose of immunoglobulin is $2 \mathrm{~g} / \mathrm{kg}$ per day. One time per day for three days. For symptomatic signs still do not fade away, $8 \mathrm{mg} / \mathrm{kg}$ methylprednisolone and $1 \mathrm{~g} / \mathrm{kg}$ immunoglobulin are used each time per day and continue for two days.(4) For child patients with bad circulation, take 10-20 mL/kg, 20\% albumin diluted to $5 \%$ per time or 10-20 mL/kg plasma for alternative. (5) Child patients with intracranial hypertension, added mannitol $0.5-1.0 \mathrm{~g} / \mathrm{kg}$, were injected with 20-30min intravenous injection every 4-8h. Adjust the time and dosage according to the condition. Ten-day is one course of treatment. It needs one course of treatment. 
Experimental group: The therapeutic treatment is as same as the western medicine group. Add Shujin Jiannao granule in the third day of treatment (Consisted by $30 \mathrm{~g}$ astragalus mongholicus, $20 \mathrm{~g}$ red peony, $3 \mathrm{~g}$ lumbricus, $6 \mathrm{~g}$ Ligusticum wallichii, $6 \mathrm{~g}$ peach kernel, $15 \mathrm{~g}$ of pericarpium zanthoxyli, full angelica, chicken vines, the root of red-rooted salvia, and lycopodium clavatum each).Take the Shujin Jiannao granule orally after dissolvement in warm water, one time per day. Seven-day is one course of treatment and take them continuously for one course of treatment.

Take Zhuang medicine of medicated thread moxibustion for external use. Acupuncture the points of Zusanli, Shenmen, Baihui and special points of Zhuang medicine, one time per day. Ten-day is one course of treatment. One course of treatment is needed.

Take records of the time for the temperature recover to normal of the two groups and the time of maculopapule disappearance on hands, feet and oral cavity and herpes escharosis.

\section{The level detection of serum S100B,NSE and CRP}

After hospitalized for $12 \mathrm{~h}$ and treated for one course of treatment, the western medicine group and experimental group were draw venous blood respectively. Took venous blood during health checkup of control group. Cryopreserved the blood sample in minus seventy degree and detected the index below respectively.

Took $2 \mathrm{~mL}$ blood,centrifuge by $1000 \mathrm{r} / \mathrm{min}$ for $10 \mathrm{~min}$ in $4{ }^{\circ} \mathrm{C}$, and separate the serum. The serum S100B and NSE levels were determined by the double antibody sandwich ELISA method. The CRP level was detected by the automatic analyzer. The number of WBC was detected by hemocytometer. The kit was provided by Beijing Biotechnology Co., Ltd.The test procedure was carried out according to the kit specification.

\section{The Criterion of therapeutic evaluation}

The Technical Guide for the Diagnosis and Treatment of HFMD (Ministry of Health, 2010) Cure: The body temperature becomes to normal. The maculopapule on hands, feet and mouth disappear and the herpes scabbed with no liquid exudation. Improvement:hypothermia, macular eruption reduced, most of the herpes scab. Inefficiency: There is no significant change in body temperature and no significant change in clinical symptoms. The Total efficiency $=$ (healing number+ effectiveness number $) /$ total number.

\section{Statistical method}

Data information was analyzed with SPSS 17. 0 software and the metering data was represented by the mean plus or minus standard deviation. Comparison before and after intra-group treatment, the paired $t$ test was used for normal distribution. If it doesn't fit the normal distribution, rank sum test was used for it. Comparison between groups, t-test was used when the variance was equal and the calibration t-test is used if the variance was unequal. The data comparison was tested by $\mathrm{x} 2$.The difference was statistically significant by $\mathrm{P}<0.05$.

\section{Result}

\section{The total effectiveness rate comparison of two groups}

Sixteen cases were cured in western group , taking up 25.8\%.Thirty-nine cases were improved, taking up $62.9 \%$.Seven cases were ineffective, taking up $11.3 \%$. Twenty cases were cured in experimental group, taking up $32.3 \%$.Forty cases were improved, taking up $64.5 \%$. Two cases were ineffective, taking up $3.2 \%$. The total effectiveness rate was $96.8 \%$. The total effectiveness rates between the two groups were statistically significant $(\mathrm{P}<0.05)$. See Table 1 .

Table 1. The total Effectiveness Rate Comparison of the Two Groups ( $n, \%)$

\begin{tabular}{llllll}
\hline Groups & Cases & Cure & \multicolumn{1}{l}{ Improvement } & Ineffectiveness The total effectiveness rate \\
\hline western group & 62 & $16(25.8)$ & $39(62.9)$ & $7(11.3)$ & $55(88.7)$ \\
experimental group & 62 & $20(32.3)$ & $40(64.5)$ & $2(3.2)$ & $60(96.8) \star$
\end{tabular}

Remark:Compared with western group, ${ }^{\star} P<0.05$. 


\section{The comparison of average disappearance time of the signs on two groups}

After treatment, the time of body temperature becoming to normal, the time of herpes disappearance on hands, feet and oral cavity and the time of herpes scab were all short than western group. Comparison with western group, the difference was statistically significant $(\mathrm{P}<0.05)$. See Table 2 .

Table 2. The Comparison of Average Disappearance Time of the Signs on Two Groups(Day)

\begin{tabular}{lcccc}
\hline Groups & Cases & body temperature becoming to normal maculopapule disappearance herpes scab \\
\hline Western group & 62 & $3.8 \pm 0.9$ & $4.2 \pm 0.8$ & $4.6 \pm 0.5$ \\
Experimental group & 62 & $2.2 \pm 0.6^{\triangle}$ & $2.1 \pm 0.6^{\triangle}$ & $2.8 \pm 0.3^{\triangle}$
\end{tabular}

Remark:Compared with western group, ${ }^{\triangle} P<0.05$.

\section{The level detection of serum S100B,NSE and CRP and counting result of WBC}

Comparison with control group, the level of serum S100B,NSE and CRP of the western group and experimental group were increased when they were hospitalized. The difference was statistically significant $(\mathrm{P}<0.05$ or $\mathrm{P}$ $<0.01)$. After treatment, the level of serum S100B,NSE and CRP decreased in western group and experimental group. There was no statistically significant difference compared with control group $(\mathrm{P}>0.05$ on average).After treatment, compared with groups in the same admission day, the level of serum S100B,NSE and CRP decreased in western group and experimental group. There was statistically significant difference $(\mathrm{P}<0.05$ or $\mathrm{P}<0.01)$. After treatment, the level of serum S100B,NSE and CRP decreased in western group and experimental group. There was no statistically significant difference $(\mathrm{P}>0.05$ on average $)$. See Table 3 .

Table 3. The Level Detection of Serum S100B,NSE and CRP and Counting Comparative Table of WBC

\begin{tabular}{|c|c|c|c|c|c|}
\hline Groups & Cases & Time & $\mathrm{S} 100 \mathrm{~B}(\mu \mathrm{g} / \mathrm{L})$ & $\mathrm{NSE}(\mathrm{ng} / \mathrm{mL})$ & $\mathrm{CRP}(\mathrm{mg} / \mathrm{L})$ \\
\hline \multirow[t]{2}{*}{ The western group } & 62 & Admission day & $1.24 \pm 0.18^{\triangle}$ & $50.21 \pm 5.45^{\triangle \Delta}$ & $11.23 \pm 2.54^{\triangle \Delta}$ \\
\hline & & After treatment & $0.28 \pm 0.03^{\mathbf{\Delta}}$ & $9.12 \pm 0.82^{\Delta \Lambda}$ & $1.03 \pm 0.12^{\wedge}$ \\
\hline
\end{tabular}

The Experimental groups

62

$$
\begin{array}{llll}
\text { Admission day } & 1.26 \pm 0.19^{\triangle} & 49.12 \pm 5.05^{\triangle \Delta} & 12.39 \pm 2.68^{\triangle \Delta} \\
\text { After treatment } & 0.16 \pm 0.04^{\Delta \Delta} & 8.06 \pm 0.71^{\Delta \Delta} & 0.86 \pm 0.08^{\Delta \Delta}
\end{array}
$$

Compared with control group, ${ }^{\triangle} P<0.05,{ }^{\triangle \triangle} P<0.01$; Compared with prior treatment, ${ }^{\mathbf{\Delta}} P<0.05,{ }^{\mathbf{\Delta}} P<0.01$.

\section{Discussion}

HFMD is a common infectious disease in pediatrics in recent years, with wide epidemic areas. The main pathogenesis is the infection of intestinal virus EV71 and CoxA16. Patients with fever and maculopapule and herpes emerging on hand, foot, mouth and buttocks as the main performances. Severe patients are often involved in nerves, organs or systems such as respiration and circulation ${ }^{[2,3]}$.For the treatment of HFMD, the main measures are expectant treatment, support, control infection and anti-virus etc. The main clinical type of HFMD combined with central nervous system damage is aseptic meningitis. The studies have shown that S100B and NSE are the sensitive index of reflecting virus EV71 and CoxA16 infection nervous system after-brain damage degree ${ }^{[4,5]} \cdot \mathrm{S} 100 \mathrm{~B}$ is usually found in astrocytes of the central nervous system. NSE is mainly found in nerve cells and neuroendocrine cells. In case of inflammation and virus infection, the function damage of blood brain barrier, S100B and NSE enter peripheral blood through damaged blood brain barrier ${ }^{[6,7]}$. The level increased in peripheral blood, the more serious of inflammatory injury and virus infection, the higher level of peripheral blood. It indicates the judge value of the level of serum S100B and NSE on HFMD order of severity. It provides basis for the study of therapeutic schedules and intervening measures on control effect of nervous system virus infectionon of HFMD on the molecular level.

Inducing inflammatory response by virus infection and immune response, therefore, alleviating inflammatory injury 
is a main aspect for the treatment of HFMD ${ }^{[8,9]}$. The previous studies have shown that CRP is the landmark of systemic inflammatory reaction and also the sensitive index of judging the order of severity. The level of CRP increases significantly when the body were infected or inflamed. The level of CRP will decrease when the inflammation got controlled ${ }^{[10,11]}$.

On this study, the western group takes standard western medicine therapeutic schedule and the experimental group takes therapeutic schedule of combining traditional Chinese and western medicine. Take notice of the post-treatment, the level of serum of S100B, NSE and CRP are both decreased. The difference is statistically significant. It is shown that the two therapeutic schedules both control the nervous system infection of HFMD and recover the level of S100B and NSE. It is also shown that the two therapeutic schedules are both effective on inflammation of HFMD.

The Shujin Jiannao granule adopted on the experimental group, rooted from the Buyang Huanwu Decoction of Qingren Wang, a famous doctor from Qing dynasty and proved recipe for the treatment of pediatric nervous system from Dong Tingyao, a famous pediatric doctor. With the function of promoting blood circulation to remove blood stasis and relaxing the muscles and tendons, dredging blocked channels.Precise treatment effect on nervous system infectious diseases $^{[12-14]}$. Medicated thread moxibustion is conventional therapy of Zhuang medicine. On the therapy of Zhuang medicine, the effect mechanism of medicated thread moxibustion is that point moxibustion can dredge the channel and stimulate the human body to be positive. It is similar to improve the immune function of body. To exorcise the physical or invisible toxins from the body. Dredging "dragon road" and "fire road", smoothing water channel, air passage and auns, the sky, earth and people are in sync, promoting the recondition of body ${ }^{[15-17]}$. Analysis from clinical efficacy results, the total effective rate is high. The time of body temperature becoming to normal, the time of maculopapule on hands, feet and oral cavity disappearance and the time of herpes scab are short than the western medicine group. It proves that conventional treatment combined with Shujin Jiannao granule and medicated thread moxibustion, combined treatment of traditional Chinese medicine and western medicine, is superior to single conventional treatment. To provide reference and discussion for the treatment of HFMD.

\section{References}

1. Wiesmann M, Missler U, Gottmann D, et al. Plasma S100B protein concentration in healthy adults is age-and sexindependent. Clin Chem 1998; 44(5): 1056-1058.

2. Liu N, Jiang Y, Li Y, et al. Study on animal model of qi stagnation blood stasis syndrome. Chinese journal of experimental Chinese medicine 2018; 24(01): 217-226.

3. Chen P, Lin C, Yang J. The contrastive analysis of the therapeutic effect of moxibustion and fire needle therapy in treating postherpetic neuralgia was analyzed. JCTM 2012; 27(07): 1847-1849.

4. Ministry of Health of the People's Republic of China, Guide for the diagnosis and treatment of HFMD. International Journal of Respiration 2010; 30( 24): 1473-1475.

5. Shu Huang, Tong Chen, Ping Zhao. Serum S100B protein determination and significance of HFMD in children with viral encephalitis. Journal of Radioimmunology 2012; 25(6): 714-715.

6. Donato R, Cannon BR, Sorci G, et al. Functions of S100 proteins. Curr Mol Med 2013; 13(1): 24-57.

7. Li J, Liu R, Shan R. Serum S100B protein and neuronal specific enolase predicted the severity of HFMD. Zhongguo Dang Dai Er Ke Za Zhi 2017; 19(2): 182-187.

8. Liao J. The value analysis of infection indicators in early diagnosis of severe HFMD. Chinese and foreign medical research 2017; 15(2): 49-50.

9. Chen Z.The detection significance of serum NSE and S100B in children with severe HFMD. Medical theory and practice 2017 ; 30(4): 586-588.

10. Ran G. Analysis of the detection significance of immunoglobulin and hypersensitive c-reactive protein in children with HFMD. Guideline of China Medicine 2017; 15(5): 29-30.

11. Zhou G. The discussion of levels changes of serum cytokines and immunoglobulin in children with different degrees of foot and mouth disease. International Journal of Laboratory Medicine 2017; 38(6): 799-804.

12. Gao J, Chen G, He Q, et al. Rule analysis of Chinese medicine treatment on qi stagnation blood stasis syndrome. Journal of Chinese Materia Medica 2017; 42(01): 187-191.

13. An Y, Wang J, He Q, et al. Study on the characteristics of symptoms of qi stagnation blood stasis syndrome based on modern literature. World Journal of Integrative Medicine 2015; 10(02): 272-274.

14. Ren X, Lu Y, Guo X, et al. Discussion on the relationship between Yin deficiency syndrome and neuroendocrine network. Henan Traditional Chinese Medicine 1998; 1998(06): 338-339+400.

15. Tan X, Liu H, Wang L, et al. 30 cases of postherpetic neuralgia treated with medicated thread moxibustion and surround needling current treatment. Journal of Clinical Acupuncture and Moxibustion 2009; 25(04): 33-34.

16. Quan X. The clinical study on the treatment of acute stage of hepatic herpes zoster by the moxibustion. Traditional Chinese Medicine University Of Guangzhou, 2017.

17. Teng H, Li F, Jiang X, et al. Normative technical study of the treatment of wind-toxic disease by medicated bath medicated thread moxibustion therapy of Zhuang medicine. Journal of External Therapy of Traditional Chinese Medicine 2009; 18(05): 3-5. 